\title{
Computational Design of Molecular Properties: Spotlight on Accuracy and Tuning
}

\author{
Clémence Corminboeuf ${ }^{\star}$
}

\begin{abstract}
The Laboratory for Computational Molecular Design at ISIC devises original and accurate methodologies to establish, in silico, key structure-property relationships of large chemical systems with particular emphasis on those associated with $\pi$-conjugated framework. Herein, we discuss two specific focuses of our activities: i) the development of accurate formalisms based on Kohn-Sham density functional theory to achieve quantitative results for the energies and geometries of extended systems featuring weak interactions and ii) the introduction of schemes to probe and tune the effect of intra- and intermolecular charge transfer on molecular properties. The proposed methodologies are ideally designed to tackle and resolve some of today's relevant aspects associated with the properties of $\pi$-conjugated molecules, such as identifying relationships resulting in high stacking capacities, proposing more stable alternative topologies to large acenes, and analyzing the course of reaction involving assemblies of $\pi$-conjugated molecules.
\end{abstract}

Keywords: Density functional theory · Molecular properties · Structure-property relationships

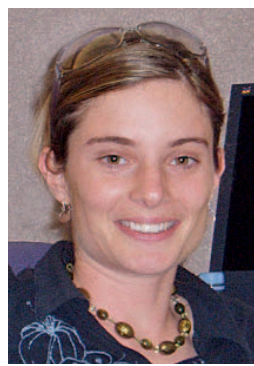

\section{Introduction}

The Laboratory for Computational Molecular Design was established in September 2007, headed by Sandoz Family Foundation Assistant Professor Clémence Corminboeuf. The overarching laboratory objective is applying and developing computational-based techniques for establishing key structure-property relationships of extended systems and designing novel functional molecules and materials. Particular emphasis is placed on properties associated with $\pi$-conjugated frameworks featuring interactions which are ubiquitous in chemistry. The interactions of $\pi$-conjugated systems play, for instance, key roles in the field of organic electronics and gas storage materials. Additionally, such interactions are responsible for the stabilization of DNA, and the binding between proteins and drugs. In pursuit of our objectives, a two-pronged approach is used (Fig. 1). The first includes developing and benchmarking accurate Kohn-Sham densi-

${ }^{*}$ Correspondence: Prof. C. Corminboeuf Laboratory for Computational Molecular Design Institut des Sciences et Ingénierie Chimiques Ecole Polytechnique Fédérale de Lausanne $\mathrm{CH}-1015$ Lausanne

E-mail: clemence.corminboeuf@epfl.ch

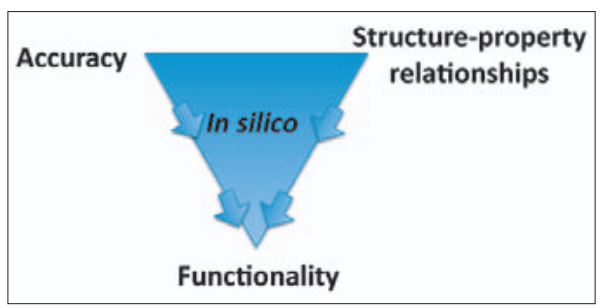

Fig. 1. Spotlight of LCMD's research.

ty functional theory-based methodologies to achieve quantitative results for the energies and geometries of large-sized molecules. Secondly, we introduce schemes to probe and tune the effect of intra- and intermolecular charge transfer on molecular properties. The following gives a brief overview of two aspects of our current ongoing research.

\section{Accuracy of Kohn-Sham Density Functional Theory}

Density functional theory (DFT) now serves as the primary workhorse of many computational/theoretical chemistry groups due to its combination of accuracy and speed. Despite its widespread use, important shortcomings are known. For instance, DFT cannot accurately describe interactions between non-overlapping densities. This result is illustrated simply by the energy associated with the sandwich benzene dimer, which is established via both experiment ${ }^{[1]}$ and high-level computation $^{[2]}$ to have an interaction energy of $1.7 \mathrm{kcal} / \mathrm{mol}$, but is either unbound or too weakly bound with standard density functionals. ${ }^{[3]}$ Similar energetic problems are also seen for seemingly simple hydrocarbon compounds, ${ }^{[4]}$ where DFT fails to reproduce the energy associated with intramolecular interactions.

As our laboratory is concerned with the accurate description of extended electronic systems and their associated properties, overcoming these errors is crucial. We demonstrated, in 2009, that inter- and intramolecular weak interactions could be corrected jointly by applying a dispersion correction tailored for weak intramolecular interactions. ${ }^{[5]}$ This simple remedy consists in accounting for the missing interactions by adding a posteriori an attractive energy term summed over all atom pairs in the system. The damped atom pairwise additive London-dispersion expression is highly valuable for introducing weak longrange attraction in molecules and soft-matter, at minimal computational cost. ${ }^{[6]} \mathrm{A}$ significant shortcoming associated with fixed empirical dispersion coefficients (used in our earlier schemes $\left.{ }^{[5,7]}\right)$ is the lack of dependency on the electronic structure. The derivation of accurate atomic dispersion coefficients dependent on the molecular environment represents an attractive alternative. Thus our more recent scheme ${ }^{[8]}$ derives system-dependent dispersion coefficients (Eqns (1) and (2)), computed according to Becke and Johnson's exchangehole dipole moment (XDM) formalism, based on the idea that the fluctuating dipole moments responsible for dispersion are related to the dipole arising from the exchange hole and its reference electron. ${ }^{[9]}$ 
The advantage of this development is the dependence of the coefficients (i.e. $C_{2 n}^{i j}$ ) on both the density and oxidation state of an atom:

$$
E_{d i s p}=\sum_{i=2}^{N_{a t}} \sum_{j=1}^{i-1} f_{2 n}\left(b R_{i j}\right) \frac{C_{2 n}^{i j}}{R_{i j}^{2 n}}
$$

where $N$ is the number of atoms in the system. To ensure robustness and general applicability, we introduced a genuine damping factor $f_{2 n}\left(b R_{i j}\right)$ with the general form of the Tang and Toennies function ${ }^{[10]}$ (Eqn. (2)), depending on Hirshfeld populations and atomic ionization energies (or free atomic polarizability), with $b$ being a damping function that serve to attenuate the correction at short internuclear distances (see ref [8a] for details).

$$
f_{2 n}(x)=1-\exp (-x) \sum_{k=0}^{2 n} \frac{x^{k}}{k !}
$$

Our most recent scheme presents an additional advantage that is the considerable simplification in the computations and implementation of the density-dependent dispersion coefficients. Instead of the Becke and Johnson's XDM formalism, ${ }^{[9 f]}$ which is relatively complex and difficult to implement, we proposed to model the dispersion coefficients (i.e. $C_{2 n}^{i j}$ ) by a generalized gradient-type approximation to the exchange hole dipole moment formalism. ${ }^{[11]}$ Our robust and efficient GGAbased density-dependent dispersion correction, $\mathrm{dDsC}$, is remarkably accurate for a broad range reactions and complexes, applicable to all elements of the periodic table and easily combined with every electronic structure methods (Figs 2 and 3). The $\mathrm{dDsC}$ scheme ${ }^{[12]}$ provides the ideal platform methodologies for describing the structures and energies of large-sized molecules such as those targeted in our current applications ${ }^{[13]}$ and for deciphering the physical principles governing their interactions. ${ }^{[14]}$

\section{Structure-Property Relationships}

Understanding the nature of inter- and intramolecular interactions and delineate their effect on molecular properties is essential, not only for establishing structure-property relationships, but also for the rational design of novel functional molecules. With this perspective, LCMD has devised a quantum chemical method enabling the direct probing and tuning of electron delocalization (i.e. intramolecular charge transfer) effects on the properties of molecules. The proposed methodology first constructs a specific resonance (i.e.
Lewis) structure, in which conjugative interactions are 'disabled' by using the simplest variant of the valence bond theory, that is the block-localized wavefunction (BLW) method. ${ }^{[15]}$ BLW first defines the intermediate electron-localized state selfconsistently at the Hartree-Fock or KohnSham density functional theory levels. Proper computations of the molecular (response) properties are then performed on both the standard (delocalized) structures and those with 'non-interacting' (localized) double bonds.

Whereas the approach was initially implemented for the computations of NMR chemical shifts (Fig. 4), [16] the formalism has been extended to other response properties such as $J$-coupling constants, ${ }^{[17]}$ first and second hyperpolarizabilities ${ }^{[18]}$ and molecular scalar fields. ${ }^{[19]}$
Recent illustrative applications of the methodology served to clarify abnormal magnetic and electronic properties of $\pi$-(hyper)conjugated systems and solve long-standing questions in organic chemistry. ${ }^{[16,17,19]}$ The peculiar electronic structure of norbornene was, for instance, elucidated by the direct comparison of its NMR properties (e.g. chemical shift and ${ }^{1} J$ coupling constants) with those of its reference analogue with a localized double bond. The BLW-NMR analysis ${ }^{[17]}$ of norbornene gives direct evidence for the manifestation of a density redistribution toward an incipient retro-Diels-Alder reaction (Fig. 5).[20] In other words, this analysis is supportive of a fascinating picture that is the fingerprint of the transition state in the ground state properties. This electronic phenomenon that is impossible

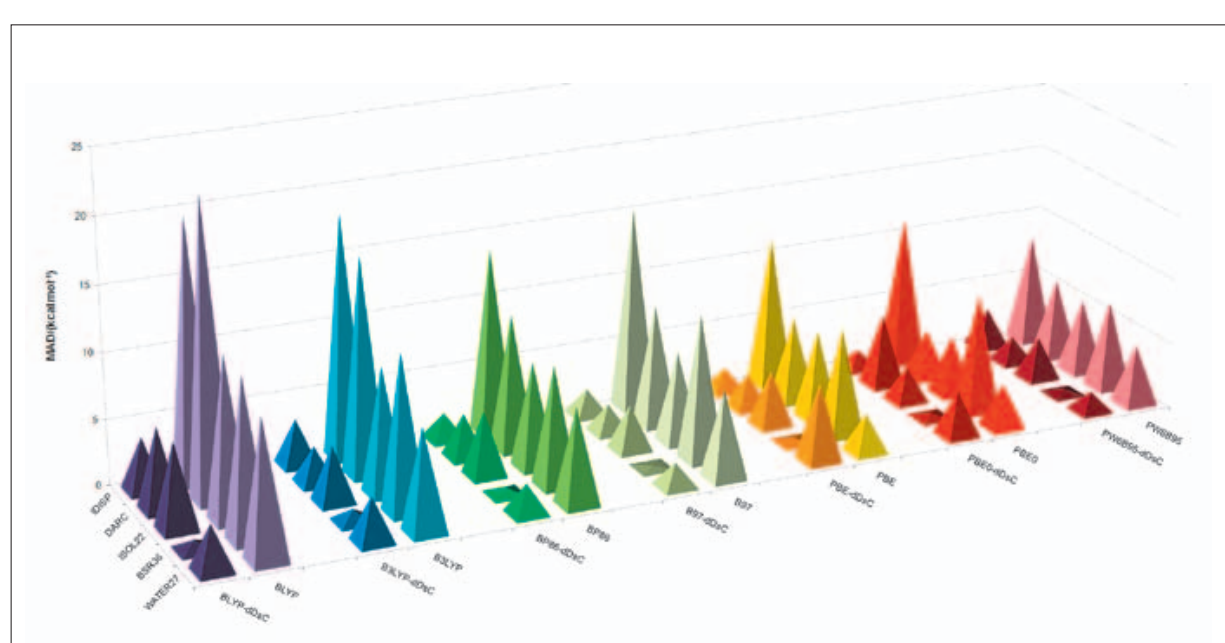

Fig. 2. Mean absolute deviations for reaction energy test sets taken from the database for general main group thermochemistry, kinetics, and non-covalent interactions (for details on the test sets see http://toc.uni-muenster.de/GMTKN/GMTKN30/GMTKN30main.htm/). Comparisons of the performance of non-corrected and dispersion corrected (dDsC) density functionals, using a quadruple- $\xi$ basis set.

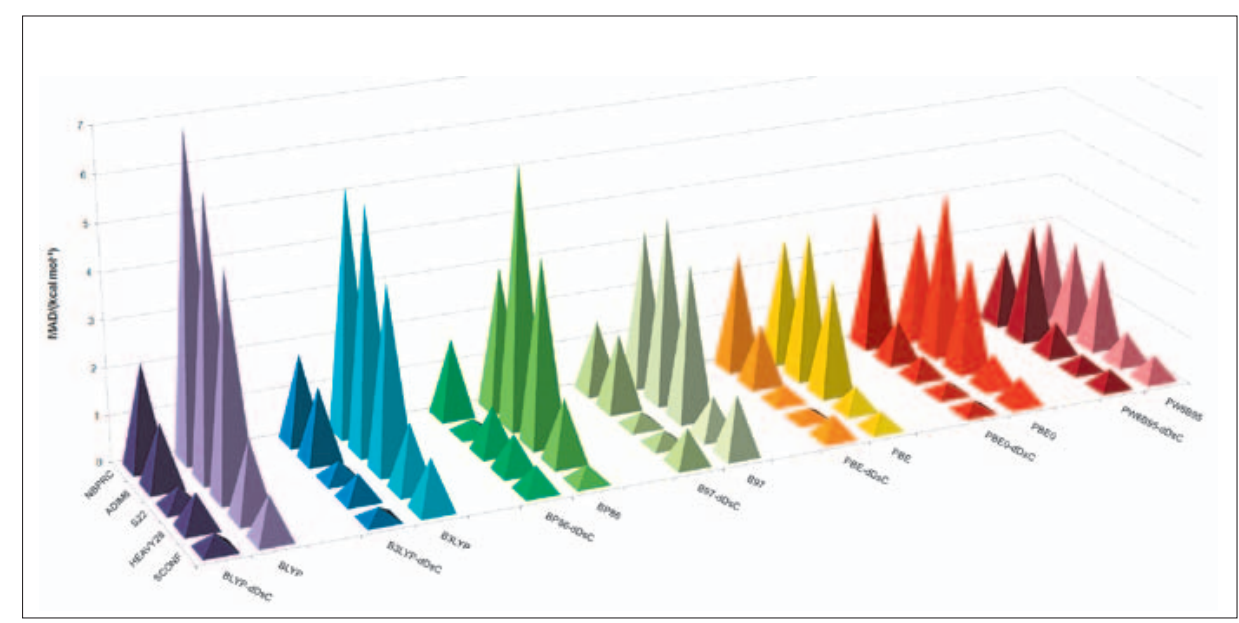

Fig. 3. Mean absolute deviations for binding energies of intermolecular complexes and sugar conformational energies taken from the database for general main group thermochemistry, kinetics, and non-covalent interactions (for details on the test sets see http://toc.uni-muenster.de/GMTKN/ GMTKN30/GMTKN30main.html. Comparisons of the performance for non-corrected and dispersion corrected (dDsC) density functionals, using a quadruple- $\xi$ basis set. 


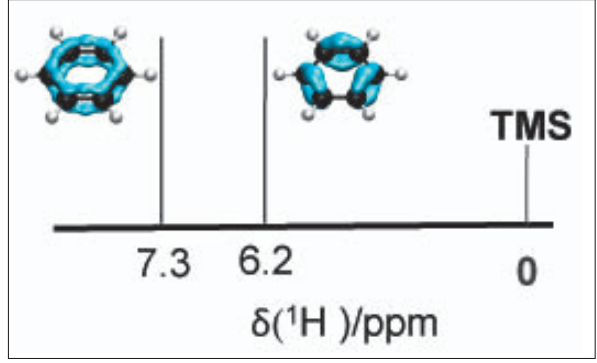

Fig. 4. NMR $\delta\left({ }^{1} H\right)$ chemical shifts for benzene (left) and 1,3,5-cyclohexatriene (1b). The isosurfaces represent the localized orbital locators of benzene and 1,3,5-cyclohexatriene. Chemical shifts are computed at the (BLW)PW91/IGLO-III level. (See ref. [15] for more details).

to demonstrate experimentally can be generalized to other pericyclic reactions (e.g. Cope). ${ }^{[21]}$

Multiple efforts to extend and validate the robustness and the fields of applicability of the current formalism are underway. In addition, special emphasis is placed on the combination ${ }^{[14]}$ and applications of both schemes (i.e. dDsC and BLW-based methods), which are ideally designed to tackle and resolve some of today's relevant aspects associated with the properties of $\pi$-conjugated molecules (e.g. identifying relationships resulting in high stacking capacities, proposing more stable alternative topologies to large acenes, analyzing the course of reaction involving assemblies of $\pi$-conjugated molecules).

Other aspects of LCMD's research, not elaborated herein, include the derivation and quantification of chemical concepts using computational means, ${ }^{[4 \mathrm{~g}, 22]}$ and the analysis of tools for the prompt characterization of potential energy surfaces. ${ }^{[23]}$

\section{Conclusions and Outlooks}

To summarize, LCMD has recently delivered accurate and innovative methodologies that allow theory and computation to obtain fundamental insights into structure-property relationships of extended systems especially those carrying a $\pi$-conjugated framework. Our simple GGA-based model for computing densitydependent dispersion coefficients has led to the inclusion of the missing dispersion interactions into standard density functional at low computational cost. Our resulting density-dependent correction, $\mathrm{dDsC}$, that has proven to be highly accurate for describing various intermolecular complexes and reaction energies, is now used routinely in our laboratory and implemented in several quantum chemistry codes. The computation of the molecular properties of resonance structures have also been shown

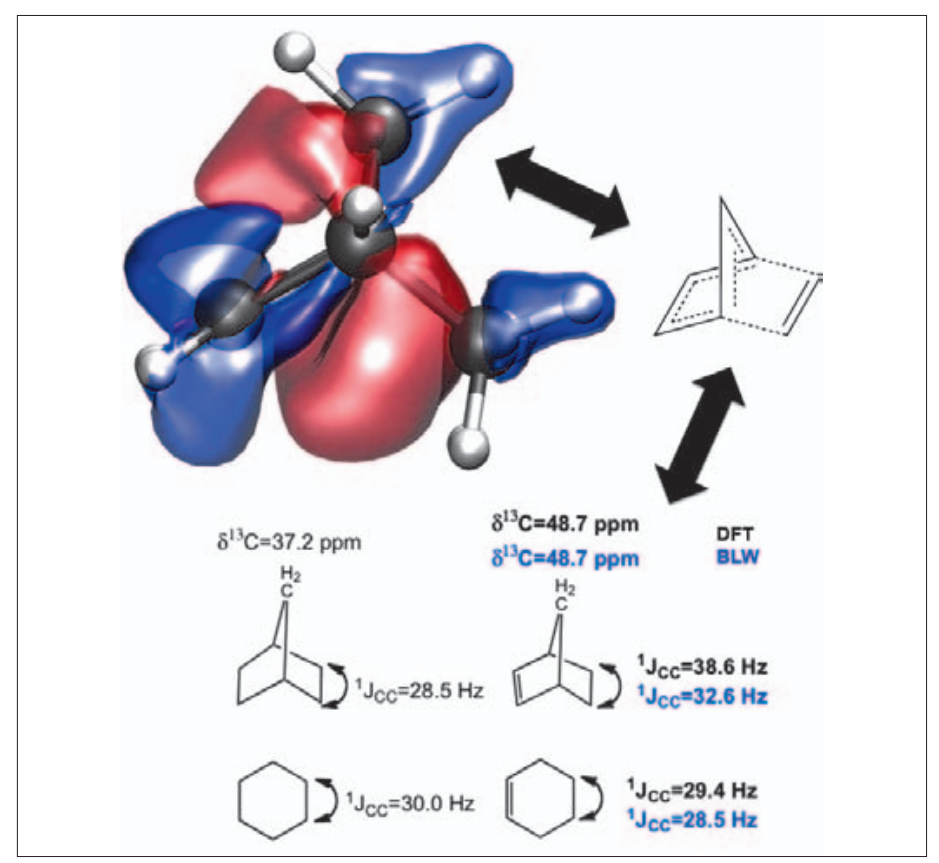

Fig. 5. Electron density difference, $\delta\left({ }^{13} \mathrm{C}\right)$ chemical shifts and ${ }^{1} \mathrm{~J}$ coupling constants of norbornene and localized norbornene (see ref. [17] for details). to offer a unique strategy for establishing relevant guidelines inaccessible otherwise. Relying on these schemes, our current research efforts should result in the design of $\pi$-conjugated molecular systems optimized with respect to their properties.

\section{Acknowledgments}

LCMD acknowledges the Sandoz family foundation, the Swiss NSF grant 200021 $121577 / 7$ and EPFL for financial support. C. C. warmly thanks all former and current members of LCMD.

\section{Received: June 24, 2011}

[1] P. M. Felker, P. M. Maxton, M. W. Schaeffer, Chem. Rev. 1994, 94, 1787.

[2] M. O. Sinnokrot, C. D. Sherrill, J. Phys. Chem A 2004, 108, 10200.

[3] For the performance of various density functional for many weakly bound complexes see: E. R. Johnson, R. A. Wolkow, G. A. DiLabio, Chem. Phys. Lett. 2004, 394, 334.

[4] a) S. Grimme, Angew. Chem. Int. Ed. 2006, 45, 4460; b) C. E. Check, T. M. Gilbert, J. Org. Chem. 2005, 70, 9828; c) M. D. Wodrich, C. Corminboeuf, P. v. R. Schleyer, Org. Lett. 2006, 8, 3631; d) P. R. Schreiner, Angew. Chem. Int. Ed. 2007, 46, 4217; e) M. D. Wodrich, C. Corminboeuf, J. Phys. Chem. A 2009, 113, 3285 ; f) S. N. Steinmann, M. D. Wodrich, C. Corminboeuf, Theor. Chem. Acc. 2010, 127, 429; g) J. F. Gonthier, M. D. Wodrich, S. N. Steinmann, C. Corminboeuf, Org. Lett. 2010, 12, 3070 .

[5] S. N. Steinmann, G. Csonka, C. Corminboeuf, J. Chem. Theory Comput. 2009, 5, 2950.

[6] a) Q. Wu, W. Yang, J. Chem. Phys. 2002, 116, 515; b) S. Grimme, J. Comput. Chem. 2006, 27 , 1787.

[7] M. D. Wodrich, D. F. Jana, P. v. R. Schleyer, C. Corminboeuf, J. Phys. Chem. A 2008, 112, 11495.

[8] a) S. N. Steinmann, C. Corminboeuf, J. Chem. Theory Comput. 2010, 6, 1990; b) S. N. Steinmann, C. Corminboeuf, Chimia 2011, 65, 240.

[9] a) E. R. Johnson, A. D. Becke, J. Chem. Phys 2005, 123, 024101; b) A. D. Becke, E. R.
Johnson, J. Chem. Phys. 2005, 123, 154101; c) A. D. Becke, E. R. Johnson, J. Chem. Phys. 2005, 122, 154104; d) A. D. Becke, E. R. Johnson, J. Chem. Phys. 2006, 124, 014104; e) E. R. Johnson, A. D. Becke, J. Chem. Phys. 2006, 124, 174104; f) A. D. Becke, E. R. Johnson, J. Chem. Phys. 2007, 127, 154108; g) A. D. Becke, E. R. Johnson, J. Chem. Phys. 2007, 127, 124108 .

[10] K. T. Tang, J. P. Toennies, J. Chem. Phys. 1984, $80,3726$.

[11] S. N. Steinmann, C. Corminboeuf, J. Chem. Phys. 2011, 134, 044117.

[12] S. N. Steinmann, C. Corminboeuf, submitted.

[13] See e.g. a) A. Olaya, P. Ge, J. Gonthier, P. Pechy, C. Corminboeuf, H. H. Girault, J. Am. Chem. Soc. 2011, 133, 12115; b) S. Rochat, S. N. Steinmann, C. Corminboeuf, K. Severin, DOI: 10.1039/clcc13927d.

[14] S. N. Steinmann, C. Corminboeuf, W. Wu, Y. Mo, J. Phys. Chem. A 2011, 115, 5467.

[15] a) Y. Mo, S. D. Peyerimhoff, J. Chem. Phys. 1998, 109, 1687; b) Y. Mo, J. Gao, S. D Peyerimhoff, J. Chem. Phys. 2000, 112, 5530; c) Y. Mo, J. Chem. Phys. 2003, 119, 1300.

[16] S. N. Steinmann, D. F. Jana, J. I.-C. Wu, P. v. R. Schleyer, Y. Mo, C. Corminboeuf, Angew. Chem. Int. Ed. 2009, 48, 9828.

[17] S. N. Steinmann, P. Vogel, Y. Mo, C. Corminboeuf, Chem. Comm. 2011, 47, 227.

[18] S. Gex, 'Understanding the NLO Properties of Push-Pull Chromophores using the BLW approach', Master Thesis, EPFL, 2011.

[19] S. N. Steinmann, Y. Mo, C. Corminboeuf, themed issue: Phys. Chem. Chem. Phys. 2011, DOI: $10.1039 / \mathrm{c} 1 \mathrm{cp} 21055 \mathrm{f}$.

[20] G. R. Jones, S. Caldarelli, P. Vogel, Helv. Chim. Acta 1997, 80, 59.

[21] D. F. Jana, C. Corminboeuf, in preparation.

[22] M. D. Wodrich, J. F. Gonthier, S. N. Steinmann, C. Corminboeuf, J. Phys. Chem. A 2010, 114, 6705.

[23] F. Avaltroni, C. Corminboeuf, J. Comput. Chem. 2011, 32, 1869 